\title{
A Dinâmica das Funções de Taper dos Modelos de Ormerod, Demaerschalk e Leite e Garcia
}

\author{
Raylan Ramos de Sa \\ Departamento de Engenharia Florestal, UFT, Gurupi, TO \\ Rasdony Klaiver Figueredo Sousa $\left.\right|^{2}$ \\ Departamento de Engenharia Florestal, UFT, Gurupi, TO \\ Cibele Cristina Trinca Watanabe $\beta$ \\ Departamento de Engenharia de Bioprocessos e Biotecnologia, UFT, Gurupi, TO \\ Ricardo Augusto Watanabe \\ Departamento de Matemática Aplicada-IMECC, UNICAMP, Campinas, SP
}

Resumo. As funções de taper são modelos biomatemáticos que visam descrever a forma e o afilamento do diâmetro do fuste das árvores. Tais funções desempenham papel central para o cálculo de biomassa das árvores e sortimentos florestais. Por este motivo, o ajuste das funções de taper implica obter uma boa aproximação ou melhora na estimativa dos multiprodutos madereiros: diâmetros, alturas e volumes parciais, totais e comerciais de troncos de árvores. O objetivo deste trabalho é o estudo da dinâmica de três modelos de taper os quais são denominados modelo de Ormerod, Demaerschalk e Leite e Garcia.

Palavras-chave. Funções de Taper, Ajuste de Funções de Taper, Processos Estocásticos

\section{Introdução}

As funções de taper são modelos biomatemáticos que visam descrever a forma e o afilamento do diâmetro do fuste das árvores [1, 2, 4]. As funções de taper são aplicadas na determinação de biomassa das árvores, isto é, através da determinação do volume do fuste das árvores é possível estimar três características fundamentais: o diâmetro em qualquer ponto do fuste, a altura no fuste em que se encontra um determinado diâmetro e o volume entre dois pontos quaisquer no fuste. Tais parâmetros permitem calcular diversos multiprodutos das árvores. Consequentemente, as funções de taper são imprescindíveis para estudos nas áreas de biologia, engenharia florestal e indústria extrativista.

Vale ressaltar que o estudo do diâmetro ao longo de um fuste também é motivado por modelos biológicos que visam estudar a geometria do tronco das árvores [3]. Por esta razão, existem na literatura as funções de taper ditas incompatíveis, em que a função de

\footnotetext{
${ }^{1}$ raylangpi2015@gmail.com

${ }^{2}$ rasdony@hotmail.com

${ }^{3}$ cibtrinca@yahoo.com.br

${ }^{4}$ ricardoaw18@gmail.com
} 
taper revela uma boa estimativa para o perfil e subseções da árvore porém o cálculo do volume total, via funções de taper, é contraditório; e existem as funções de taper ditas compatíveis, em que o cálculo de volume e subseções da árvore, via funções de taper, é concordante [2]. O estudo conduzido por Demaerschalk [5,6] revela que há compatibilidade quando o volume total, obtido pela soma dos volumes de todas as seções determinadas através de modelos de taper, é idêntico ao volume definido pela equação de volume (integral da função de taper em relação á medida da altura do fuste da árvore).

Via de regra as funções de taper compatíveis são funções que dependem da altura total da árvore $(H)$ (estimativa tabelada segundo o gênero da árvore), de parâmetros de referência da árvore: altura $\left(H_{r}\right)$ de referência e diâmetro de referência $\left(d_{a p}\right)$ (parâmetros em que é realizada a poda ou estudo da árvore, em geral são valores constantes por uma questão de uniformidade para cada gênero de árvore) e de fatores de taper $(\beta)$ (responsáveis por descrever a geometria do fuste da árvore. Estes parâmetros são obtidos empericamente e tabelados segundo o gênero da árvore ). Por este motivo as funções de taper são adaptadas para incluir termos adicionais de diâmetro e/ou altura do fuste. São exemplos de funções de taper compatíveis os modelos de Kozak (1969) [7], Demaerschalk (1972) [5, Ormerod (1973) 8] e Leite e Garcia (2001) 9]. Sendo que o modelo de Leite e Garcia é inspirado no modelo de Demaerschalk [9].

Em [10] os modelos de Kozak, Ormerod e Demaerschalk foram modificados a fim de acrescentar parâmetros estocásticos. Tal estudo é motivado por observações biológicas de que a pluviosidade (parâmetro estocástico) afeta diretamente o crescimento das árvores [2]. Segundo [10] é possível analisar a variação de diâmetro, altura e volume ao longo de um fuste frente à tais perturbações estocásticas.

Motivado por [10], este trabalho aborda questões relacionadas à dinâmica dos modelos de taper. O objetivo deste trabalho é discutir e estudar a dinâmica de três modelos de taper: modelo de Ormerod, Demaerchalk e Leite e Garcia por meio de equações diferenciais. Na seção 2 iremos descrever as principais hipóteses de tais modelos bem como descrever seus parâmetros. Na seção 3 iremos introduzir uma dinâmica capaz de descrever a evolução temporal dos modelos. Na seção 4 iremos simular e analisar tal dinâmica sobre o ponto de vista dos parâmetros biológicos e na seção 5 iremos discutir os resultados obtidos.

\section{Funções de Taper: Modelo de Ormerod Modificado, De- maerschalk e Leite e Garcia}

No contexto da área instrumental de mensuração florestal as funções de taper são tradicionalmente obtidas empiricamente, isto é, coletados os dados biológicos é realizado algum método de regressão para estimar os parâmetros (vide [11] para maiores detalhes e para métodos de estimativa envolvendo redes neurais). Desta forma, sabendo-se o gênero da árvore estudada é possível estimar o diâmetro, altura, coeficientes de taper e tempo de poda [4].

Todavia esta abordagem não leva em conta a dinâmica da evolução do crescimento das árvores. Esta seção visa estabelecer os fundamentos dos modelos de Ormerod, Demaerschalk e Leite e Garcia com o objetivo de abordar tais modelos do ponto de vista dinâmico 
na seção 4 .

Os modelos de Ormerod [8], Demaerschalk [6] e Leite e Garcia [9] consideram o crescimento da árvore uniforme e homogêneo. Tais modelos são dados, respectivamente, pelas seguintes expressões:

$$
\begin{gathered}
d=\operatorname{dap}\left[\frac{(H-h)}{\left(H-H_{r}\right)}\right]^{\beta_{1}} e^{\beta_{2} T_{x}}+\epsilon \\
d=\left[10^{\beta_{0}} d a p^{\left(\beta_{1}-1\right)} H^{\beta_{2}}(H-h)^{\beta_{3}} \exp \left(\beta_{4} T_{x}\right)\right]+\epsilon, \\
d=\left[10^{\left(\beta_{0}\right)} d a p^{\beta_{1}-1} H^{\beta_{2}}(H-h)^{\beta_{3}} \exp \left(\beta_{4} \frac{T_{x}}{d a p}\right)\right]+\epsilon
\end{gathered}
$$

onde os parâmetros são:

- $h$ é a altura, medida em metros;

- $d$ é o diâmetro comercial, com ou sem casca, medido em centímetro, na altura h;

- $H$ é a altura total, medida em metros;

- $H_{r}$ é a altura de referência, medida em metros, usualmente é adotado a convenção de 1,30 metros;

- $d_{a p}$ é o diâmetro de referência, medido em centímetro, obtido na altura de referência $H_{r}$

- $T_{x}=0,1$ para diâmetro com casca e sem casca, respectivamente;

- $\beta_{i}$, para $i=0,1,2,3,4$, são denominados coeficientes de taper. São parâmetros de regressão e, com excessão de $\beta_{4}$ em (3) que possui dimensão de metro, são parâmetros adimensionais;

- $\epsilon$ é o erro aleatório, onde $\epsilon \sim N\left(0, \sigma^{2}\right)$.

Os coeficientes de taper são responsáveis por descrever a geometria da fuste da árvore [3]. Uma análise dimensional revela que para os modelos (2) e (3) temos $\beta_{1}+\beta_{2}+\beta_{3}=2$. Note que as equações (1), (2) e (3) revelam que $d=0$ ocorre para $h=H$, em outras palavras, o diâmetro da árvore é decrescente. Para a equação (1) $d=d a p$ precisamente quando $h=H_{r}$, isto é, o diâmetro de referência é obtido na altura de referência. Ainda para o modelo (1), temos que caso $\beta_{1} \leq 1$ então a forma do tronco será parabólico e caso $\beta_{1} \geq 1$ então a forma do tronco será neiloidal [8].

Ao longo deste trabalho iremos fazer as seguintes hipótese simplificadoras:

1. Por hipótese, $H_{r}$ e $d_{a p}$ são parâmetros fixos para cada espécie de árvore;

2. O diâmetro e a altura da árvore estão sujeitas a uma dinâmica de evolução temporal;

3. Os parâmtros de taper não evoluem com o tempo, isto é, são coeficientes fixos; 
4. desconsiderar os inerentes processos aleatórios.

Observe ainda que através da seguinte mudança de variavel adimensional $y=\frac{d}{d_{a p}} \mathrm{e}$ $x=\frac{h}{H}$ é possível concatenar as equações (1), (2) e (3) da seguinte forma:

$$
y=\alpha_{i}(1-x)^{\gamma_{i}}
$$

onde para a equação (1) temos $\alpha_{1}=\frac{H^{\gamma_{1} \exp \left(\beta_{2} T x\right)}}{\left(H-H_{r}\right)^{\beta_{1}}}$ e $\gamma_{1}=\beta_{1}$, para a equação (2) temos $\alpha_{2}=10^{\beta_{0}} \operatorname{dap}^{\left(\beta_{1}-2\right)} H^{\beta_{2}} H^{\gamma_{3}} \exp \left(\beta_{4} T_{x}\right)$ e $\gamma_{2}=\beta_{3}$ e para a equação (3) temos $\alpha_{3}=$ $10^{\left(\beta_{0}\right)} d a p^{\beta_{1}-2} H^{\beta_{2}} H^{\gamma_{3}} \exp \left(\beta_{4} \frac{T x}{d a p}\right)$ e $\gamma_{3}=\beta_{3}$. Pela análise dimensional dos coeficientes de taper, temos que todos os parâmetros $\alpha_{i}$ são adimensionais.

Por meio da equação (4), introduziremos na seção 3 uma dinâmica de crescimento da altura da árove.

\section{Metodologia}

Segundo [2,4] os modelos de Ormerod, Demaerschalk e Leite e Garcia são aplicáveis ás árvores de gênero Pinus e Eucalyptus, portanto faz sentido comparar a dinâmica da evolução de cada modelo de taper.

Por este motivo, em uma primeira aproximação, iremos considerar que a velocidade de crescimento do volume da árvore $(V)$ é constante $(K)$ e que tanto o raio da seção transversal da árvore quanto a altura da árvore são funções do tempo. Desta forma obtemos,

$$
\frac{d V}{d t}=k=2 \pi r \frac{d r(t)}{d t} h+\pi r^{2} \frac{d h(t)}{d t}
$$

Observe que a derivada de (7) em relação ao tempo para $\gamma_{i}$ nulo é constante nula e para $\gamma_{i}$ não nulo é dada por:

$$
\frac{d y}{d t}=-\alpha_{i} \gamma_{i}(1-x)^{\gamma_{i}-1} \frac{d x}{d t}
$$

As função de taper (1), (2), (3) fornecem o raio em função do tempo $(r(t)=d(t) / 2)$. Considerando a condição inicial $x(0)=H_{r} / H$, utilizando a mudança de variável exposta na seção 2, as equações (4) e (6) é possível obter a seguinte equação diferencial ordinária:

$$
\left\{\begin{array}{l}
\frac{d x(t)}{d t}=\frac{\tilde{K}(1-x(t))^{-2 \gamma_{i}+1}}{-2 \gamma_{i} x(t)+1} \\
x(0)=\frac{H_{r}}{H}
\end{array}\right.
$$

onde o parâmetro adimensional

$$
\tilde{K}=\frac{K}{4 \pi H \alpha_{i}^{2} d_{a p}^{2}}
$$


representa a taxa do crescimento volumétrico para determinada função de taper, onde o fator $H \alpha_{i}^{2} d_{a p}^{2}$ possui unidade de volume e está associado ao cálculo do volume da árvore através de funções de taper.

A equação (7) é uma equação diferencial ordinária (EDO) de primeira ordem não-linear capaz de descrever a evolução da altura da árvore para os modelos de (1), (2), (3). Na seção 4 iremos simular a equação (7) para diferentes parâmetros biológicos e discutir tais resultados.

\section{Simulações e Resultados}

A literatura fornece diversos valores para a altura, diâmetro e coeficientes de taper para diversos gêneros de árvores $1,2,2,5,7,8$. Para efeito de simulação iremos considerar apenas as árvores da espécie Pinus elliottii. Segundo 2 tal espécie de pinheiro apresenta altura média total de $H=26 m$, diâmetro de referência $d_{a p}=0,2 m$ e crescimento volumétrico anual médio a partir da idade de maturação de $V=0,052 \mathrm{~m}^{3} /$ ano. Sobre os coeficientes de taper, para esta espécie de árvore com esta altura e diâmetro, temos os valores de $\beta_{0}=-0,6552, \beta_{1}=1,476038, \beta_{2}=-0,65520$ e $\beta_{3}=1.179162$. Por meio de tais valores é possível calcular os parâmetros $\alpha_{i}$ bem como a taxa de crescimento volumétrico $\tilde{K}$. Também iremos considerar apenas o caso das árvores com casca, isto é, $T x=0$ uma vez que a ausência de casca é de ordem de grandeza desprezível.

Uma maneira de estudar a equação (7) é por meio da análise do campo de direções para diferentes valores de $\tilde{K}$ e $\gamma_{i}$ ( Figuras 1 12 3 3 4). A equação (7) revela que existem descontinuidades quando $x_{1}(t)=1$ e $x_{2}(t)=1 / 2 \gamma_{i}$ (retas verticais nas Figuras 112 3). É possível estudar a região entre tais descontinuidades. Na Figura 1 existe um efeito repulsivo nas vizinhanças de $x(0)$ até $x_{1}$ e outro efeito repulsivo para $x(t)>x_{1}$, assim para valores de $\gamma_{i}$ negativos o diâmetro decresce rapidamente como era esperado; a região $x(x)<x(0)$ não possui sentido biológico. Para valores de $\gamma_{i}$ positivos, como nas Figuras 2 e 3 , existe um efeito atrativo nas vizinhanças de $x_{2}\left(x_{0}<x(t)<x_{2}\right)$, o valor de $x_{2}$ se desloca para diferentes valores de $\gamma_{i}$ e ainda o campo de direções sofre uma inflexão devido á mudança de velocidade e direção $\left(x_{2}<x(t)<x_{1}\right)$ que é proporcional ao valor de $\tilde{K}$.

Note ainda que é possível resolver a EDO (7) explicitamente. Por exemplo para o caso particular de $\gamma_{i}=1 / 2$ temos

$$
\left\{\begin{array}{l}
\frac{d x(t)}{d t}=\frac{\tilde{K}}{1-x}, \\
x(0)=\frac{H_{r}}{H}
\end{array}\right.
$$

Cujo plano de fase está representada na Figura 4 para diferentes valores de $\tilde{K}$. Observe ainda na Figura 4 a transição de fase que ocorre a medida que o parâmetro $\gamma$ aumenta. A solução de (9) é dada por:

$$
x(t)=1-\left(1-\frac{H_{r}}{H}\right) \exp (-\tilde{K} t)
$$

Vale notar que (9) é condizente com o modelo (7) pois o limite $x(t \rightarrow 0)=H_{r} / H$ representa o valor inicial e $x(t \rightarrow \infty)=1$ representa a altura máxima. 


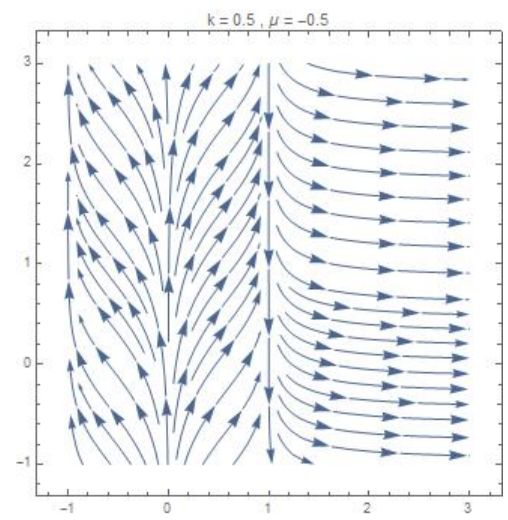

Figura 1: Modelo de Ormerod com casca refinado.

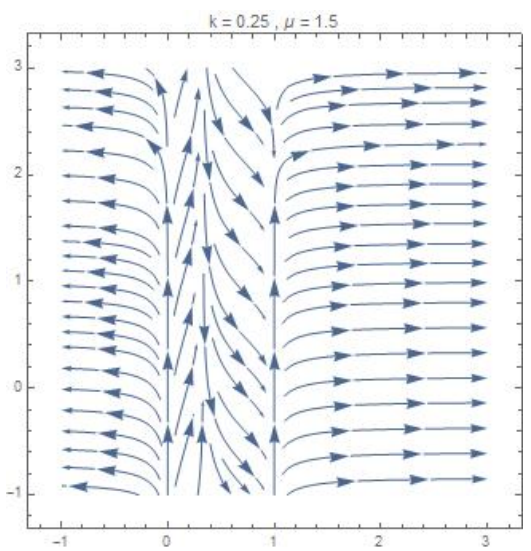

Figura 3: Modelo de Ormerod com casca refinado.

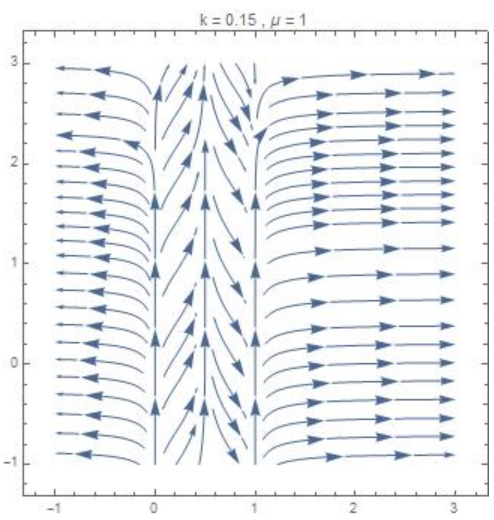

Figura 2: Modelo de Ormerod sem casca refinado.

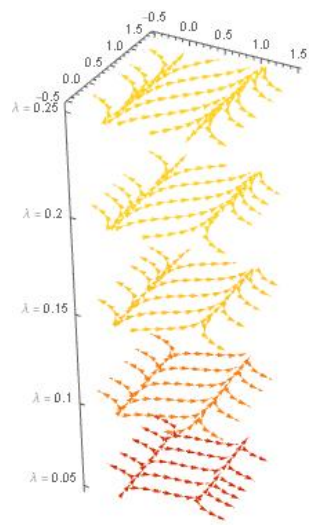

Figura 4: Planos de fase da eq. (7) para diferentes valores de $\tilde{K}$.

\section{Conclusões}

A dinâmica de crescimento da altura das árvores de gênero Pinus Eucalyptus pode ser modelada, a partir das funções de taper apresentadas na seção 2, pela equação (7). O o campo de direções de (7) revela efeitos de transição de fase em determinadas regiões, como observado na seção 4 . No contexto da área de engenharia florestal tal abordagem é inovadora pois agrega diferentes modelos e permite estudar a evolução da altura da árvore em questão em função de parâmetros biológicos implícitos nos parâmetros $\alpha_{i}$ tais como pluviosidade, qualidade do solo e quantidade de luz.

Existem limitações para tal abordagem apresentada. Por exemplo, o modelo de Kozak é um modelo polinomial e não pode ser abordado da mesma forma. Outro ponto limitante é que as hipósteses simplificadoras 3 e 4 da seção 2 não são condizentes com a realidade: a pluviosidade é um processo estocástico que interfere na forma e no crescimento da árvore. Por este motivo, em trabalhos futuros, a modelagem da dinâmica das funções de taper 
deve incorporar os parâmetros biológicos implícitos e se valer de equações diferenciais estocásticas.

\section{Agradecimentos}

O presente trabalho foi realizado com o apoio da Universidade Federal do Tocantins (UFT) e os autores agradecem ao Conselho Nacional de Desenvolvimento Científico e Tecnológico $(\mathrm{CNPq})$.

\section{Referências}

[1] J. F. Borges, Seccionamento do fuste de Pinus taeda L. para a obtenção do volume de madeira serrada através da função de forma polinomial, Dissertação de Mestrado, UFPR, 1981.

[2] J. C. C. Campos and H. G. Leite. Mensuração Florestal: perguntas e respostas. Editora UFV, Viçosa-MG, 2016. ISBN: 857269465X.

[3] R. R. Forslund. A geometrical tree volume model based on he location of the centre of gravity of the bole, Can. J. For. R., 12(2): 215-221,1982.

[4] J. A. Kershaw Jr., M. J. Ducey, T. W. Beers, B. Husch, Forest Mensuration, 5 a edição. John Wiley and Sons, New Jersey, 2016.

[5] J. P. Demaerschalk. Integrated systems for the estimation of tree taper and volume, Can. J. For. R., 03:90-94, 1972.

[6] J. P. Demaerschalk. Converting volume equations to compatible taper equations, Forest Science, 18:241-245, 1972.

[7] A. Kozak, D. D. Munro and J. H. G. Smith. Taper functions and their application in forest inventory, Forestry Chronicle, 45:278-283, 1969.

[8] D. W. Ormerod. A simple bole model, Forestry Chronicle, 49:136-138, 1973.

[9] H. G. Leite, S. L. R. Garcia. Pesquisa e desenvolvimentos em inventário, mensurações e manejo florestal na CENIBRA, Investigações Florestais, 3:49-56, 2001.

[10] de Sa, R. R. ; Santos, B. M. M. ; Rosa, P. H. L. ; Sousa, R. K. F. ; Resende, C. R. ; Trinca, C. C. ; Watanabe, R. A.Aspectos Biomatemáticos de Funções de Taper via Conceitos Estocásticos, Proceeding Series of the Brazilian Society of Computational and Applied Mathematics, volume 2, 2018. https://doi.org/10.5540/03.2018. 006.02 .0256

[11] F. A. A.M.N. Soares, E. L. Flôres, C. D. Cabacinha, G. A. Carrijo, A. C. P. Veiga. Recursive diameter prediction for calculating merchantable volume of Eucalyptus clones without previous knowledge of total tree height using artificial neural networks. Appl. Soft Comp., 12(8): 2030-2039, 2012. 